\title{
Reactivation of the Mitosis-Promoting Factor in Postmitotic Cardiomyocytes
}

\author{
Daniel A. Dätwylera Josef P. Magyar ${ }^{a}$ Christian Weikert ${ }^{a}$ \\ Lionel Wightman $^{\mathrm{b}}$ Ernst Wagner $^{\mathrm{c}}$ Hans M. Eppenberger ${ }^{\mathrm{a}}$ \\ a Institute of Cell Biology, Swiss Federal Institute of Technology, Zürich, Switzerland; 'bBoehringer Ingelheim Austria, \\ Vienna, Austria; ${ }^{c}$ Centre of Drug Research, University of München, Munich, Germany
}

\section{Key Words}

Cardiomyocytes, cultured · Cell cycle •

Mitosis-promoting factor · Adenovirus-enhanced

transferrinfection - Gene transfer · Rat

\begin{abstract}
Cardiomyocytes cease to divide shortly after birth and an irreversible cell cycle arrest is evident accompanied by the downregulation of cyclin-dependent kinase activities. To get a better understanding of the cardiac cell cycle and its regulation, the effect of functional recovery of the mitosis-promoting factor (MPF) consisting of cyclin B1 and the cyclin-dependent kinase Cdc2 was assessed in primary cultures of postmitotic ventricular adult rat cardiomyocytes (ARC). Gene transfer into ARC was achieved using the adenovirus-enhanced transferrinfection system that was characterized by the absence of cytotoxic events. Simultaneous ectopic expression of wild-type versions of cyclin B1 and Cdc2 was sufficient to induce MPF activity. Reestablished MPF resulted in a mitotic phenotype, marked by an abnormal condensation of the nuclei, histone $\mathrm{H} 3$ phosphorylation and variable degree of decay of the contractile apparatus. Although a complete cell division was not observed, the
\end{abstract}

results provided conclusive evidence that cell cycle-related events in postmitotic cardiomyocytes could be triggered by genetic intervention downstream of the G1/S checkpoint. This will be of importance to design novel strategies to overcome the proliferation arrest in adult cardiomyocytes.

Copyright $\odot 2003$ S. Karger AG, Base

\begin{tabular}{ll}
\hline KARGER & ( ) 2003 S. Karger AG, Basel \\
Fax +4161306 1234 $34-6405 / 03 / 1752-0061 \$ 19.50 / 0$ \\
$\begin{array}{l}\text { E-Mail karger@karger.ch } \\
\text { www.karger.com }\end{array}$ & $\begin{array}{l}\text { Accessible online at: } \\
\text { www.karger.com/cto }\end{array}$
\end{tabular}

Prof. Dr. H.M. Eppenberger

Institute of Cell Biology, ETH-Hönggerberg

CH-8093 Zürich (Switzerland)

Tel. +411633 33 57, Fax +4116331152

E-Mail hme@cell.biol.ethz.ch 


\section{Introduction}

The concept of maintaining contractile efficiency of a failing heart by reinduction of cardiomyocyte proliferation has spurred the investigation of cell cycle proteins in postmitotic cardiomyocytes by transgenic means. Induction of cell cycle reentry at the G1/S boundary by ectopic expression of the adenoviral oncogene E1A and the transcription factor E2F-1 led to an increase in DNA synthesis in cultured rat cardiomyocytes [Kirshenbaum and Schneider, 1995; Liu and Kitsis, 1996; Agah et al., 1997; Akli et al., 1999; von Harsdorf et al., 1999]. However, an entry into the $M$ phase leading finally into mitosis and cytokinesis could not be observed under these experimental regimes. Recent findings in transgenic mice overexpressing cyclin-dependent kinase cdk2 have further emphasized the existence of a cell cycle block at the G2/M boundary, since forced expression of cdk2 was not sufficient to reactivate gene expression of $\mathrm{cdc} 2(\mathrm{cdk} 1 / \mathrm{p} 34)$ and cyclin B1 [Liao et al., 2001]. The complex of cyclin B1/ cdc2 protein kinase, the so-called mitosis-promoting factor (MPF), is activated in eukaryotic cells, which enter mitosis [Nurse, 1990]. During heart development, mRNA and protein levels of cyclin $\mathrm{B} 1$ and $\mathrm{Cdc} 2$ are both decreasing, thus provoking a significant downregulation of MPF activity in ventricular rat cardiomyocytes shortly after birth up to the adult stage [Brooks et al., 1997; Kang et al., 1997; Poolman and Brooks, 1998].

In this study, we aimed at the functional recovery of MPF in the cardiac cell cycle by ectopic expression of cyclin B1 and cdc2. The intention was to obtain knowledge of the control patterns responsible for the arrest downstream of the G1/S transition and to find clues to overcome the block of cell-cycle progression.

So far, use of recombinant gene technology in cultured ventricular adult rat cardiomyocytes (ARC) employing standard gene transfection methods was inefficient [Rust et al., 1998] or was dependent on virus-based gene transfer systems [Kass-Eisler et al., 1993; Kirshenbaum et al., 1993; Dätwyler et al., 1999, 2001; Wei et al., 2000]. In the context of the present study, we evaluated the adenovirusenhanced transferrinfection (AVET) system representing a novel way to transfect cultured ARC without the need of active viral particles.

We present results which provide information about the reactivation of the cell cycle at the $\mathrm{G} 2 / \mathrm{M}$ boundary in cultured ARC. The in vitro system may allow extrapolation to the in vivo situation.

\section{Materials and Methods}

\section{Plasmid Constructions}

The expression vector pcDNA3.1cycB1-HA was generated in the following way: human cyclin B1 was cloned by the reverse transcription polymerase chain reaction (RT-PCR) using the QIAGEN OneStep RT-PCR Kit (QIAGEN, Hilden, Germany) from RNA extracts of synchronized HeLa cells in G2/M phase. Synchronization was accomplished by the double thymidine block method [Stein et al., 1998]. RNA isolation of synchronized HeLa cells was performed using the Trizol Reagent (Gibco, Grand Island, N.Y., USA) according to the manufacturer's protocol. Cyclin B1 was amplified and directly HA-tagged at the C-terminus end using the following primers: forward 5'-gccaccATGGCGCTCCGAGTCACCAGGAAC-3' and reverse 5'-ttaagcgtagtctgggacgtcgtatgggtaCACCTTTGCCACAGCCTTGGCTAAATC-3' (gene coding sequences are indicated by capital letters). The amplified cyclin B1 fragment was subsequently cloned into pcDNA3.1/V5/His-TOPO vector (Invitrogen, Groningen, The Netherlands) according to the manufacturer's protocol, resulting into pcDNA3.1 cycB1-HA.

To generate the double expression vector pcDNA3.1cycB1-HA/ cdc2-FLAG, the small AvrII/BstZ17I fragment of $\mathrm{pSBC} 2 \mathrm{cdc} 2$ FLAG was ligated into the large AvrII/BstZ17I fragment of pcDNA3.1cycB1-HA. pSBC2cdc2-FLAG was constructed by amplifying and FLAG-tagging the human cdc2 at the C-terminus by PCR from the template pCMVcdc2-HA (kindly provided by Dr. Bruno Amati, ISREC, Epalinges, Switzerland) using the forward primer 5'-gccaccATGGAAGATTATACCAAAATAG-3' and the reverse primer 5'-cctacttatcgtcgtcatccttgtaatcCATCTTCTTAATCTGATTGTCC-3'. The PCR fragment was subcloned into pcDNA3.1/ V5/His-TOPO leading to pcDNA3.1cde2-FLAG. The XhoI/BamHI fragment of pcDNA3.1cdc2-FLAG encoding cdc2-FLAG was subsequently cloned under the control of the SV40 promoter by ligation into the $\mathrm{BamHI} / \mathrm{SalI}$ site of pSBC-2 [Dirks et al., 1993] resulting into pSBC2cdc2-FLAG.

The double expression vector pcDNA3.1cycB1-HA/cdc2AFFLAG was cloned by inserting the cdc2AF-FLAG gene derived from pSBC2cdc2AF-FLAG into the large AvrII/BstZ17I fragment of pcDNA3.1 cycB1-HA. The vector pSBC2cdc2AF-FLAG was cloned in the same way as pSBC2cdc2-FLAG (see above). The phosphorylation site mutations (T14A, Y15F) were introduced by PCR amplification from pCMVcdc2-HA using the forward primer 5'-gccaccATGGAAGATTATACCAAAATAGAGAAAATTGGAGAAGGTGCCTTTGGAGTTGTGTATAAGG-3' and the reverse primer 5'-cctacttatcgtcgtcatcettgtaatcCATCTTCTTAATCTGATTGTCC-3'. The authenticity of all PCR fragments was confirmed by sequencing.

The reporter plasmids pHM6-lacZ and pEGFP-N3 were purchased from Roche Diagnostics (Mannheim, Germany) and Clontech Laboratories (Palo Alto, Calif., USA), respectively.

\section{Cardiomyocyte Isolation and Cultivation}

Ventricular cardiac myocytes were isolated from 6- to 8-weekold female OFA rats (BRL, Füllinsdorf, Switzerland) by retrograde perfusion of the hearts according to an established method [Eppenberger-Eberhardt et al., 1990]. Culture dishes were coated with $0.1 \%$ gelatin. ARC were cultured right after the isolation in medium containing medium M199 (Amimed, Basel, Switzerland), 10\% FCS (PAA Laboratories, Linz, Austria), $20 \mathrm{mmol} / 1$ creatine monohydrate (Sigma, St. Louis, Mo., USA) and $1 \%$ penicillin/streptomycin (Gibco, Grand Island, N.Y., USA). Proliferation of fibro- 
blasts and other proliferation-competent cells was inhibited by adding $10 \mu M$ cytosine arabinoside (ICN Biochemicals, Cleveland, Ohio, USA) to the medium. After 5 days in culture, cytosine arabinoside was omitted and the medium was supplemented with $2 \mathrm{mmol} / \mathrm{l} L$-glutamine (Amimed) to provide culture conditions that would not potentially inhibit cell cycle progression in ARC having reactivated MPF.

\section{Culture and Transfection of HEK 293 Cells}

HEK 293 cells were grown in DMEM (Amimed) supplemented with 10\% FCS (Biological Industries, Kibbutz Beth Haemek, Israel), $2 \mathrm{mmol} / \mathrm{l} \mathrm{L}$-glutamine (Amimed), 1\% penicillin/streptomycin (Gibco) in a humidified atmosphere at $37^{\circ} \mathrm{C}$ with $6 \% \mathrm{CO}_{2}$.

Transfection of HEK 293 cells was performed by the calcium phosphate precipitation method according to a modified protocol [Westfall et al., 1997].

\section{Transfection of Cultured ARC using the AVET System}

Gene transfection of cultured ARC was achieved by using the AVET system consisting of transferrin-poly $(L)$ lysine conjugates (Tf$\mathrm{pL}$ ) with an average chain length of 250 lysine residues, streptavidinpolylysine conjugates (STAV-pL) and biotinylated, psoralen-inactivated adenovirus (AdV) dl1014 [Cotten et al., 1992; Wagner et al., 1992]. Cultured ARC were generally transfected 6-8 days after the isolation procedure. For optimal transfection efficiency of ARC, $3 \mu \mathrm{g}$ STAV-pL in $100 \mu \mathrm{l} \mathrm{HBS}(150 \mathrm{mmol} / \mathrm{l} \mathrm{NaCl}, 10 \mathrm{mmol} / \mathrm{l} \mathrm{HEPES}$, pH 7.3) were mixed with $1.2 \times 10^{10}$ inactivated AdVdl1014 in $100 \mu \mathrm{HB}$ and incubated for $30 \mathrm{~min}$ at room temperature. After adding $3 \mu \mathrm{g}$ of plasmid DNA in $150 \mu \mathrm{l} \mathrm{HBS}$, a further 30-min incubation step followed at room temperature. The mixture was combined with $3 \mu \mathrm{g}$ Tf-pL in $150 \mu \mathrm{l} \mathrm{HBS}$ and incubated for $30 \mathrm{~min}$ before the transfection mix was added dropwise onto ARC cultures. Afterwards, the dishes were centrifuged at $100 \mathrm{~g}$ for $5 \mathrm{~min}$, supporting a rapid concentration of the transfection complexes on the cell surface. Cultivation medium was replaced $4-16 \mathrm{~h}$ after the transfection and analysis was performed at the indicated time points.

Quantitative determination of the gene transfer efficiency using the AVET system was conducted by transfecting pEGFP-N3. At least 500 cells were counted in each experiment. Experiments were performed in triplicate. The results are expressed as mean \pm standard error of the mean.

\section{Immunofluorescence Analysis and Microscopy}

Cells were fixed in 3\% paraformaldehyde in PBS for $15 \mathrm{~min}$, washed with PBS, permeabilized in $0.2 \%$ Triton X-100/PBS for 12 min and preincubated with $0.1 \%$ bovine serum albumin in PBS for $20 \mathrm{~min}$. The cells were then incubated with primary antibodies for 2-3 $\mathrm{h}$ at room temperature. After washing with PBS, secondary antibodies were added for $1 \mathrm{~h}$. Fluorescently labeled cells were analyzed using a Zeiss Axiophot fluorescence microscope equipped with Neofluar objectives (Zeiss, Feldbach, Switzerland).

\section{Immunoblotting}

HEK 293 cells were washed and scraped off into lysis buffer [0.1 mol/1 Tris buffer $\mathrm{pH} 6.8,2 \%$ sodium dodecyl sulfate (SDS), $20 \%$ glycerol and protease inhibitors]. For Western blot analysis, equal amounts of protein lysates judged by Ponceau staining were separated on a $12.5 \%$ SDS-polyacrylamide gel and transferred to a nitrocellulose membrane (Hybond ${ }^{\mathrm{TM}}-\mathrm{C}$ extra, Amersham Pharmacia Biotech, Uppsala, Sweden). Incubation with primary antibodies was done for $2 \mathrm{~h}$ at room temperature. After washing, the incubation with secondary antibodies was performed for $1 \mathrm{~h}$ at room temperature. Chemiluminescence detection was performed with SuperSignal (Pierce, Rockford, Ill., USA) according to the manufacturer's protocol.

\section{Antibodies}

The monoclonal mouse (mM) anti-myomesin antibody (clone B4; generated in this laboratory) [Grove et al., 1984] and the polyclonal rabbit anti-titin M8 antibody [Obermann et al., 1996], generously provided by Dr. M. Gautel, MPI Dortmund, were used as sarcomeric markers. The monoclonal rat anti-HA antibody (clone 3F10) was purchased from Roche Diagnostics. For the detection of the FLAG-epitope, the mM anti-FLAG M2 antibody from Sigma was used. The polyclonal rabbit anti-phospho-histone $\mathrm{H} 3$ antibody was purchased from Upstate Biotechnology (Lake Placid, N.Y., USA). Antibody staining against endogenous cyclin B1 was performed by using an $\mathrm{mM}$ anti-cyclin B1 antibody (clone GNS1; Santa Cruz, Calif., USA). The $\mathrm{mM}$ anti-cdk1/cdc2 antibody (clone 1; C12720) was obtained from Transduction Laboratories (Lexington, Ky., USA).

For immunofluorescence stainings, combinations of fluorescein isothiocyanate (FITC), cyanine-3 (Cy3) or Texas Red (TxRed), and cyanine-5 (Cy5)-conjugated secondary antibodies together with DAPI (Molecular Probes, Eugene, Oreg., USA) were used. FITC-conjugated goat anti-mouse IgG (Jackson Immuno Research, Hamburg, Germany) was used for the detection of the anti-FLAG, anti-cdk1/ cdc2, anti-cyclin B1 antibody. FITC-conjugated goat anti-rabbit IgG (Cappel Research, West Chester, Pa., USA) was used to visualize the anti-phospho-histone H3 and anti-titin M8 antibody. The TxRedconjugated goat anti-mouse IgG was used to detect the anti-myomesin antibody. The Cy3-conjugated donkey anti-rat IgG (Jackson Immuno Research) was used throughout the work to detect the antiHA antibody. The Cy5-coupled donkey anti-mouse IgG (Jackson Immuno Research) was applied to visualize the anti-myomesin, antiFLAG, and anti-cdk1/cdc2 antibody.

For immunoblotting, horseradish peroxidase (HRPO)-conjugated goat anti-mouse IgG and HRPO-conjugated rabbit anti-rat IgG (both Dako Diagnostics, Zug, Switzerland) were used as secondary antibodies.

\section{Results}

\section{Gene Delivery Mediated by the AVET System into Cultured ARC}

The AVET system had already been demonstrated in a number of experimental setups to be very useful for the transfection of various cell types [Wightman et al., 1999; Huber et al., 2000]. In cultured ARC, gene transfer by the AVET system was tested by varying the respective ratios of the AVET components, which are biotinylated psoralen-inactivated adenoviruses, STAV-pL, Tf-pL and plasmid DNA. Highest gene transfection efficiency was obtained by a transfection mix consisting of $3 \mu \mathrm{g}$ of plasmid DNA, $1.2 \times 10^{10}$ inactivated adenoviral particles, $3 \mu \mathrm{g}$ Tf-pL and 1.5-3 $\mu \mathrm{g}$ STAV-pL. An additional significant 


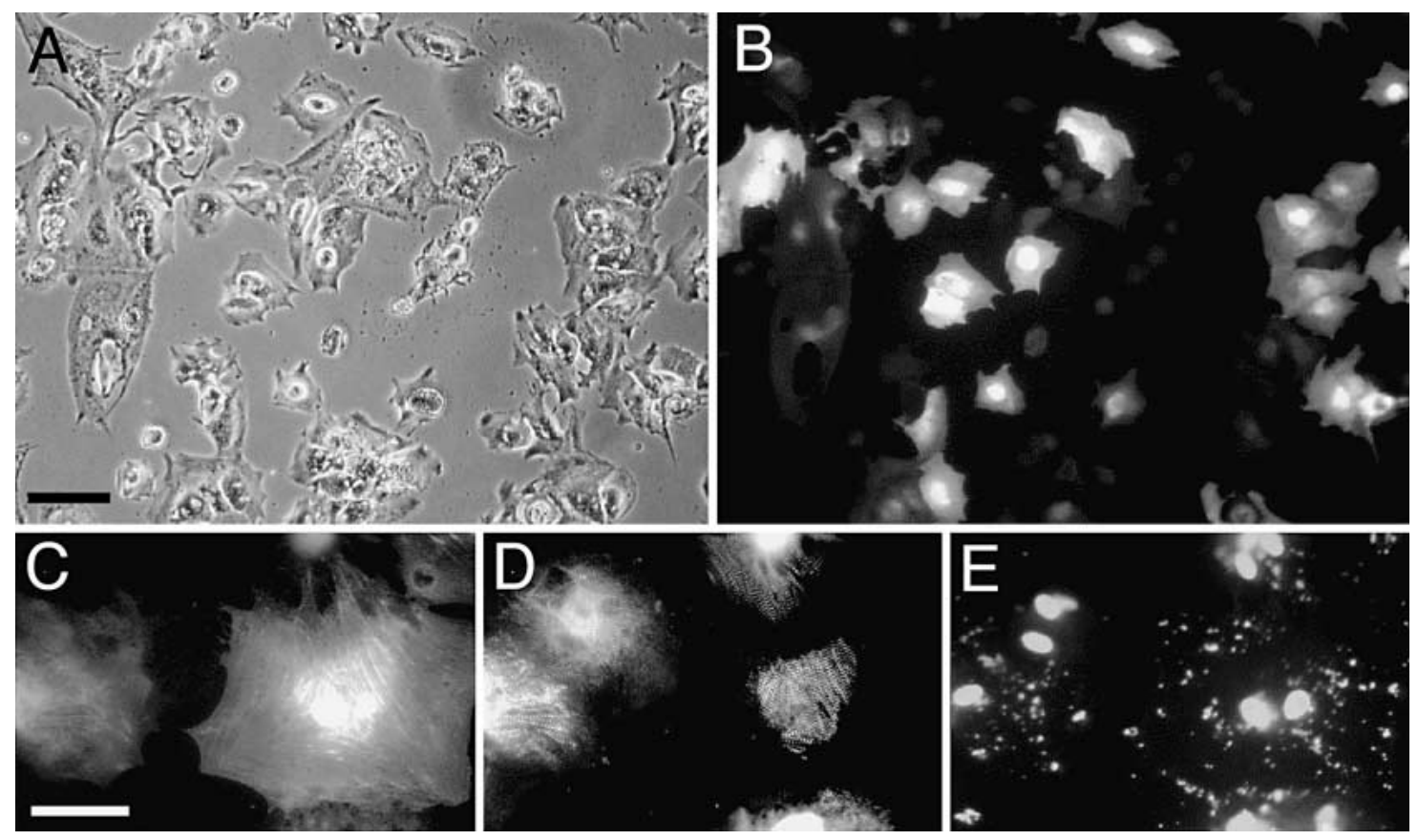

Fig. 1. EGFP expression in cultured ARC after gene transfection using the AVET system. ARC kept 7 days in culture were transfected with pEGFP-N3 and analyzed on the following days. A Phase contrast picture of cultured ARC. B EGFP fluorescence was detected in $22 \pm 6 \%$ of the cultured cells (determined from three independent transfection experiments). C-E Immunofluorescence analysis of cultured ARC. C The transgene product (EGFP) was strongly expressed in transfected ARC, tending to accumulate in the perinuclear region of the cell. D Anti-myomesin antibody staining. E DNA staining with DAPI. The AVET complexes were easily identified as the little spots distributed over transfected cells. Scale bar: $100 \mu \mathrm{m}(\mathbf{A}), 50 \mu \mathrm{m}(\mathbf{C})$.

improvement was achieved by centrifuging the whole dishes after the transfection mix had been applied to the culture dishes. Under these conditions, transfection was achieved in more than $20 \%$ of cultured ARC with pEGFP-N3, which encodes the reporter gene EGFP driven by the CMV promoter (fig. 1). Immunofluorescence analysis of untransfected cells as well as of cells expressing the transgene within the same dish allowed a valid comparison. Several days after the transfection an intact myofibrillar apparatus and no changes in the shape of nuclei were observed. Additionally, unrestricted beating activity of the transfected ARC could be demonstrated revealing an unaffected viability and also proving the low cytotoxicity of the gene transfer method used.

\section{Ectopic Expression of Cyclin B1 in Cultured ARC}

As a prelude to establish strategies for MPF activity, the presence of endogenous $\mathrm{Cdc} 2$ and cyclin $\mathrm{B} 1$ in cultured ARC was investigated (fig. 2). A weak signal was observed in single-cell analysis using immunofluorescence microscopy suggesting only a very small amount of
Cdc2 present in the nuclei of cultured ARC. No specific nuclei staining for cyclin B1 was observed in ARC as could be expected from in vivo data previously published by other groups [Brooks et al., 1997; Kang et al., 1997; Poolman and Brooks, 1998].

Being aware of the weak signal for $\mathrm{Cdc} 2$, we determined whether functional recovery of MPF in cultured ARC could be accomplished by expression of exogenous cyclin $\mathrm{B} 1$ alone in order to complement the absence of endogenous cyclin B1. Cultured ARC were transfected by using the AVET system with pcDNA3.1 cycB1-HA encoding HA-tagged cyclin B1 under the control of the CMV promoter. Analysis of nuclei and general morphologic appearance of transfected ARC was performed $72 \mathrm{~h}$ posttransfection, being sufficient to express the exogenous protein and for the detection of completed or ongoing cell cycle-related events in transfectants. The presence of the transgene product and endogenous $\mathrm{Cdc} 2$ was confirmed by anti-HA antibody and anti-Cdc2 antibody staining (fig. 3). HA-tagged cyclin B1 was diffusely distributed throughout the cytoplasm and nuclei. No obvious abnor- 
Fig. 2. Expression of MPF components in ARC kept for 9 days in culture and analyzed by immunofluorescence microscopy. Cultured ARC stained with anti-cyclin B1 antibody (A) and anti-cdk1/cdc2 antibody (B). C, D DAPI staining revealed nuclei. E, F Cardiomyocytes were identified by antibody staining against the sarcomeric protein titin that regularly resulted in additional nuclei staining. No specific staining for cyclin B1 was obvious, suggesting its absence in cultured ARC (A, C, E). Possible presence of small amounts of $\mathrm{Cdc} 2$ protein in cultured ARC was proposed by the weak signal detected in the nuclei of cultured $\operatorname{ARC}(\mathbf{B}, \mathbf{D}$, F). Scale bar: $25 \mu \mathrm{m}$.
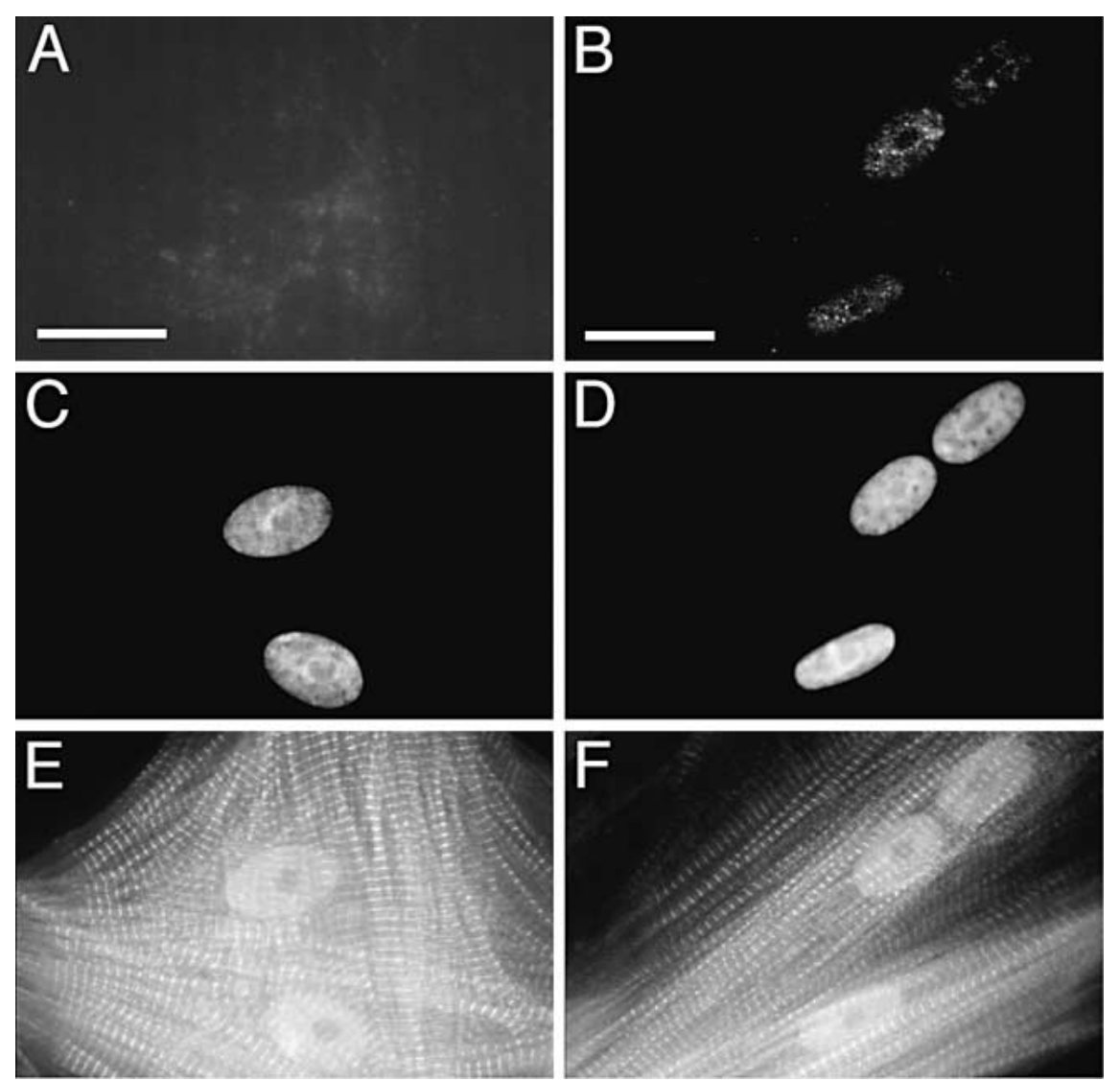

malities in cell architecture were seen in these cells compared to untransfected ones. Special attention was paid to the nuclei of transfectants in order to detect chromosomal condensation induced by an active MPF complex. No changes in chromatin structure were found; transfected ARC still exhibited interphase-like nuclei.

Instead of forced expression of cyclin B1, double expression vectors encoding both genes, namely cyclin B1 and cdc2, were subsequently employed to restore MPF activity. These MPF expression vectors, termed pcDNA3.1 cycB1-HA/cdc2-FLAG and pcDNA3.1cycB1$\mathrm{HA} / \mathrm{cdc} 2 \mathrm{AF}-\mathrm{FLAG}$, were derived from the mono-expression vectors pcDNA3.1cycB1-HA, pSBCcdc2-FLAG and pSBCcdc2AF-FLAG (fig. 4A). The latter encoded a constitutively active mutant form of $\mathrm{Cdc} 2$, termed $\mathrm{Cdc} 2 \mathrm{AF}$, which circumvents the inhibitory pathway controlled by the Wee1 kinase [Krek and Nigg, 1991]. Functionality of the MPF double expression vectors pcDNA3.1cycB1-HA/ cdc2-FLAG and pcDNA3.1cycB1-HA/cdc2AF-FLAG were checked by transient transfection into HEK 293 cells. Western blot analysis confirmed the presence of both transgene products in transfected HEK 293 cells (fig. 4B).

\section{Recovery of MPF Activity and Its Effects in ARC}

Cultured ARC were transfected with pcDNA3.1 cycB1HA/cdc2-FLAG and pcDNA3.1cycB1-HA/cdc2AFFLAG, respectively, using the AVET system. ARC were allowed to express the exogenous proteins for $72 \mathrm{~h}$ and were examined afterwards by indirect immunofluorescence analysis. The presence of the transgene products cyclin B1-HA, Cdc2-FLAG and Cdc2AF-FLAG was verified by antibody staining against the corresponding epitopes used for tagging. A small number of transfected ARC per dish expressing the MPF components was readily detectable by immunofluorescence microscopy and some striking morphologic features became evident in transfected ARC where both transgene products were clearly detectable (fig. 5). The nuclei of such transfected ARC displayed a highly compacted appearance associated with an abnormal clumped morphology. The high consistency observed $(>90 \%$, determined from several independent transfection experiments) between altered nuclei and the presence of cyclin B1-HA and Cdc2-FLAG or Cdc2AF-FLAG, respectively, indeed suggested the functional recovery of MPF in ARC by the double expression 

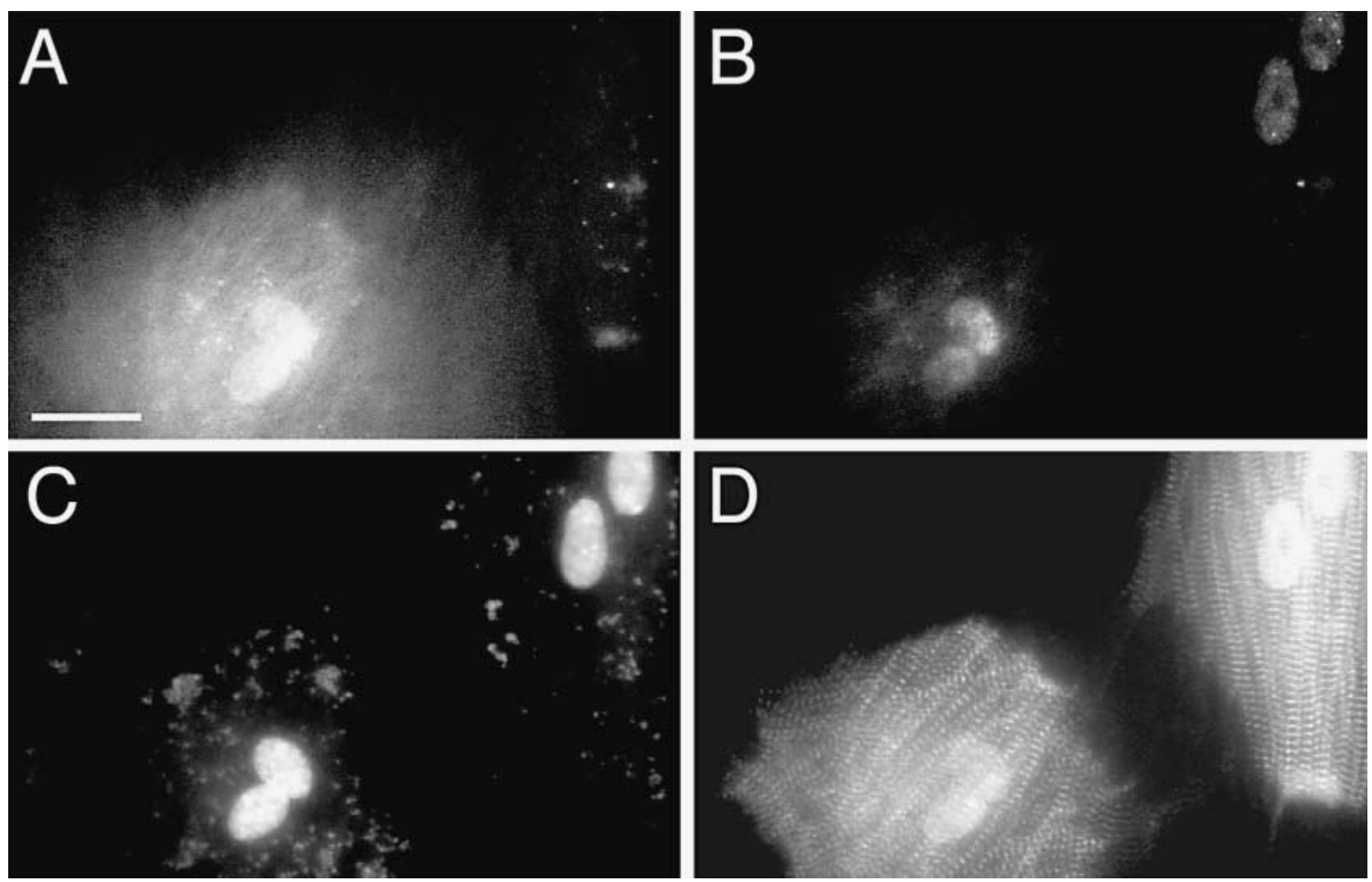

Fig. 3. Cultured ARC transfected with pcDNA3.1cycB1-HA. Immunofluorescence analysis. A-D Identical fields $72 \mathrm{~h}$ after transfection with the AVET system. A Expression of cyclin B1-HA in ARC was shown by anti-HA antibody leading to nuclei and cytoplasmic staining, and allowing the distinction between untransfected and transfected ARC. B Low expression of endogenous Cdc2 in the nuclei of cultured ARC was shown by anti-cdk1/cdc2 antibody staining. C DAPI staining showed no differences regarding the nuclei morphology between untransfected and transfected ARC in culture. The contractile apparatus was still well organized in ARC expressing cyclin B1-HA as demonstrated by staining against the sarcomeric protein titin (D). Scale bar: $25 \mu \mathrm{m}$.

vectors. A special preference to induce MPF activity could not be accredited to pcDNA3.1cycB1-HA/cdc2AFFLAG encoding the constitutively active mutant cdc2AF, since ARC transfected with pcDNA3.1cycB1-HA/cdc2FLAG encoding the wild-type version cdc2 underwent nuclei transformation with the same efficiency.

Tightly packed nuclei induced by MPF activity in ARC were frequently accompanied by a degradation of the contractile structures (fig. $5 \mathrm{G}, \mathrm{H}$ ). The degree of myofibrillar degradation in ARC transfected with pcDNA3.1cycB1-HA/cde2-FLAG and pcDNA3.1cycB1$\mathrm{HA} / \mathrm{cdc} 2 \mathrm{AF}-\mathrm{FLAG}$ was variable. It was, however, obvious that MPF activity was the direct or eventually the indirect cause of myofibrillar decay in these cells.

Mitotic chromosome condensation has been known to be strongly linked with histone $\mathrm{H} 3$ phosphorylation [Koshland and Strunnikov, 1996]. A possible correlation of phosphorylation of histone $\mathrm{H} 3$ with MPF expression was analyzed using an antibody highly specific for the phosphorylated form of the amino terminus of histone $\mathrm{H} 3$ at serine 10 [Hendzel et al., 1997]. Control transfections using pHM6-lacZ, which encoded HA-tagged lacZ, did not induce $\mathrm{H} 3$ phosphorylation (fig. $6 \mathrm{~B}, \mathrm{D}, \mathrm{F}, \mathrm{H}$ ). In contrast to nontransfected ARC, phosphorylation of histone H3 could be demonstrated in all transfected ARCexpressing MPF components and displaying nuclear condensation (fig. 6A, C, E, G).

\section{Discussion}

The difficulty to transfer genes into ARC may be responsible for the fact that only one report has described the analysis of cell cycle-regulating factors in cultured ARC by genetic means [Agah et al., 1997]. Here, the AVET system has been demonstrated to be a reliable and 


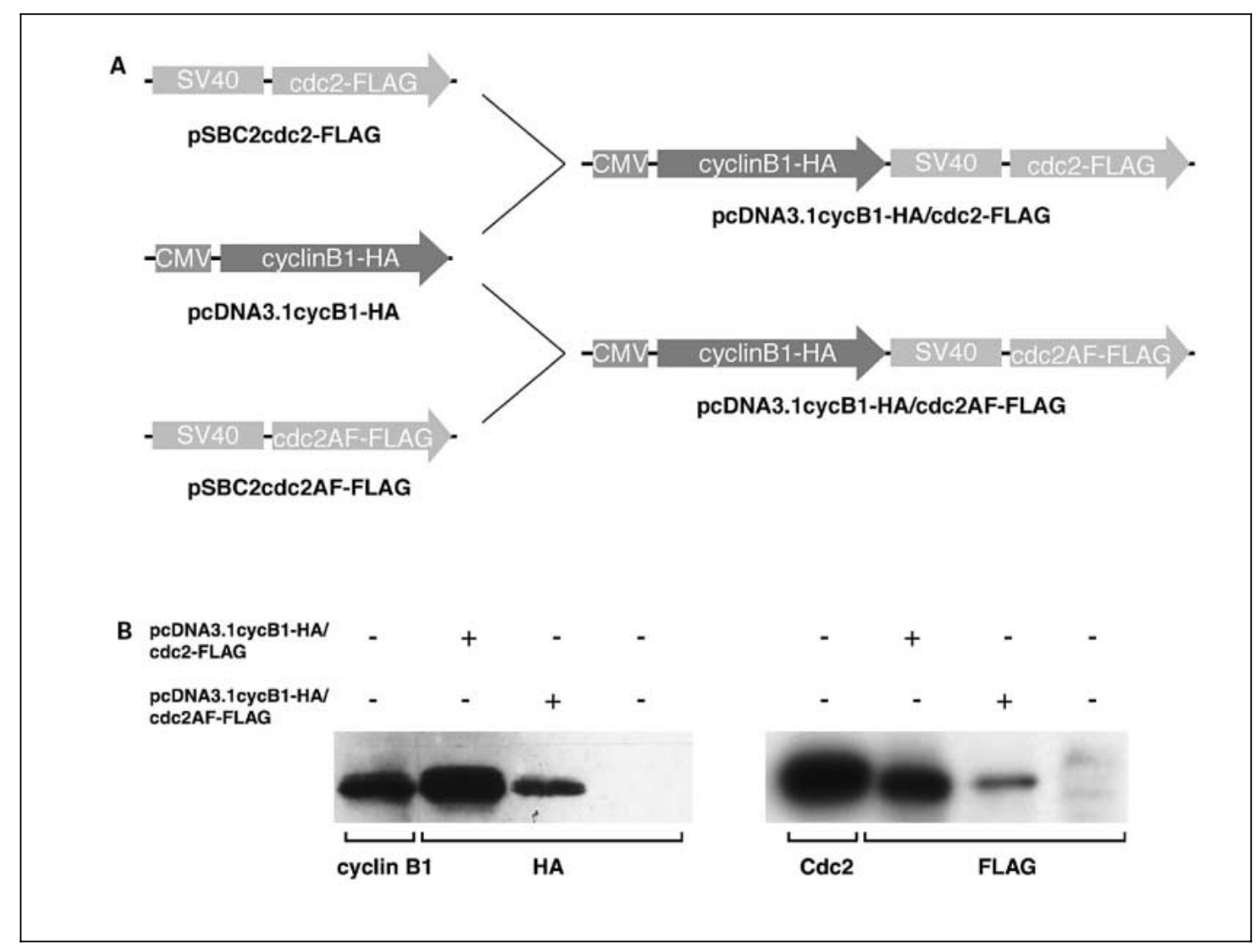

Fig. 4. Construction of mono- and double expression cassettes generated to restore MPF activity. A The vectors pcDNA3.1cycB1-HA, pSBC2cdc2-FLAG, and pSBC2cdc2AF-FLAG are precursors of the MPF expression vectors pcDNA3.1 cycB1-HA/cdc2-FLAG and pcDNA3.1 cycB1-HA/cdc2AF-FLAG. The wild-type cdc2 and cdc2AF gene, respectively, both FLAG-tagged, were put under the control of the SV40 promoter, whereas transcription of cyclin $\mathrm{B} 1-\mathrm{HA}$ is driven by the CMV promoter. Cdc2AF encodes the phosphorylation site mutant of Cdc2 (T14A, Y15F) rendering it constitutively active (see Results). B Western blot analysis of HEK 293 cells transiently transfected with the MPF vectors pcDNA3.1cycB1-HA/cdc2-FLAG and pcDNA3.1cycB1-HA/cdc2AF-FLAG. Cells were harvested $40 \mathrm{~h}$ after calcium phosphate transfection and probed for the presence of cyclin B1-HA and Cdc2-FLAG/Cdc2AFFLAG, using anti-HA and anti-FLAG antibodies, respectively. Antibody staining against endogenous $\mathrm{Cdc} 2$ and cyclin B1 served as a control for the size of the exogenous counterparts.

easier way to genetically manipulate the adult cardiac cell cycle. Gene transfection efficiency of up to $20 \%$ and more was achieved with a construct encoding the reporter gene EGFP. The implementation of the AVET system enabled also reliable statements regarding gene function in cultured ARC. Transfections performed with control vectors have ruled out the possibility of transfection-derived artifacts. Despite the rather high variations observed in transfection efficiency for different constructs, the AVET system has represented, besides the tedious microinjection, the only reliable way to deliver genes into cultured ARC without the use of active viral particles. Generally, the
AVET system may thus be attractive for a wide range of applications in biomedical cardiac research.

In primary culture systems of ARC, the tight mechanism regulating entry into the $\mathrm{S}$ phase was reported to be overcome by ectopic expression of the transcription factor E2F-1 [Agah et al., 1997], whereas control mechanisms involved in the $\mathrm{G} 2 / \mathrm{M}$ transition still remained elusive. To obtain information on the regulation of the cardiac cell cycle at the $\mathrm{G} 2 / \mathrm{M}$ site, we aimed to reestablish the activity of the MPF complex composed of cyclin B and Cdc 2 . The amount of active endogenous $\mathrm{Cdc} 2$ was eventually too low to generate enough kinase activity or, on the other 
Fig. 5. MPF recovery in ARC. Cultured ARC were transfected with the double expression vectors pcDNA3.1 cycB1-HA/cdc2-FLAG (A, C, E, G) and pcDNA3.1 cycB1-HA/cdc2AF-FLAG (B, $\mathbf{D}, \mathbf{F}, \mathbf{H})$. Immunofluorescence staining was conducted $72 \mathrm{~h}$ after transfection. Presence of the exogenous gene products cyclin B1-HA (A, B), Cdc2-FLAG (C) and Cdc2AF-FLAG (D) was confirmed by antibody staining against the corresponding tag epitopes. Nuclei were visualized by DAPI labeling (E, F). Cardiomyocytes were identified by anti-titin $(\mathbf{G}, \mathbf{H})$. Simultaneous expression of cyclin B1-HA (A, B) and Cdc2-FLAG (C)/ Cdc2AF-FLAG (D) led to chromosome condensation (E, F). The corresponding nuclei showed a reduced size with an unusual morphology that was associated with an eye-catching bright DAPI signal compared to untransfected cells (E, left side of panel). Condensed nuclei were accompanied by a mild to severe decay of the contractile apparatus $(\mathbf{G}, \mathbf{H})$. Scale bar: $10 \mu \mathrm{m}$.
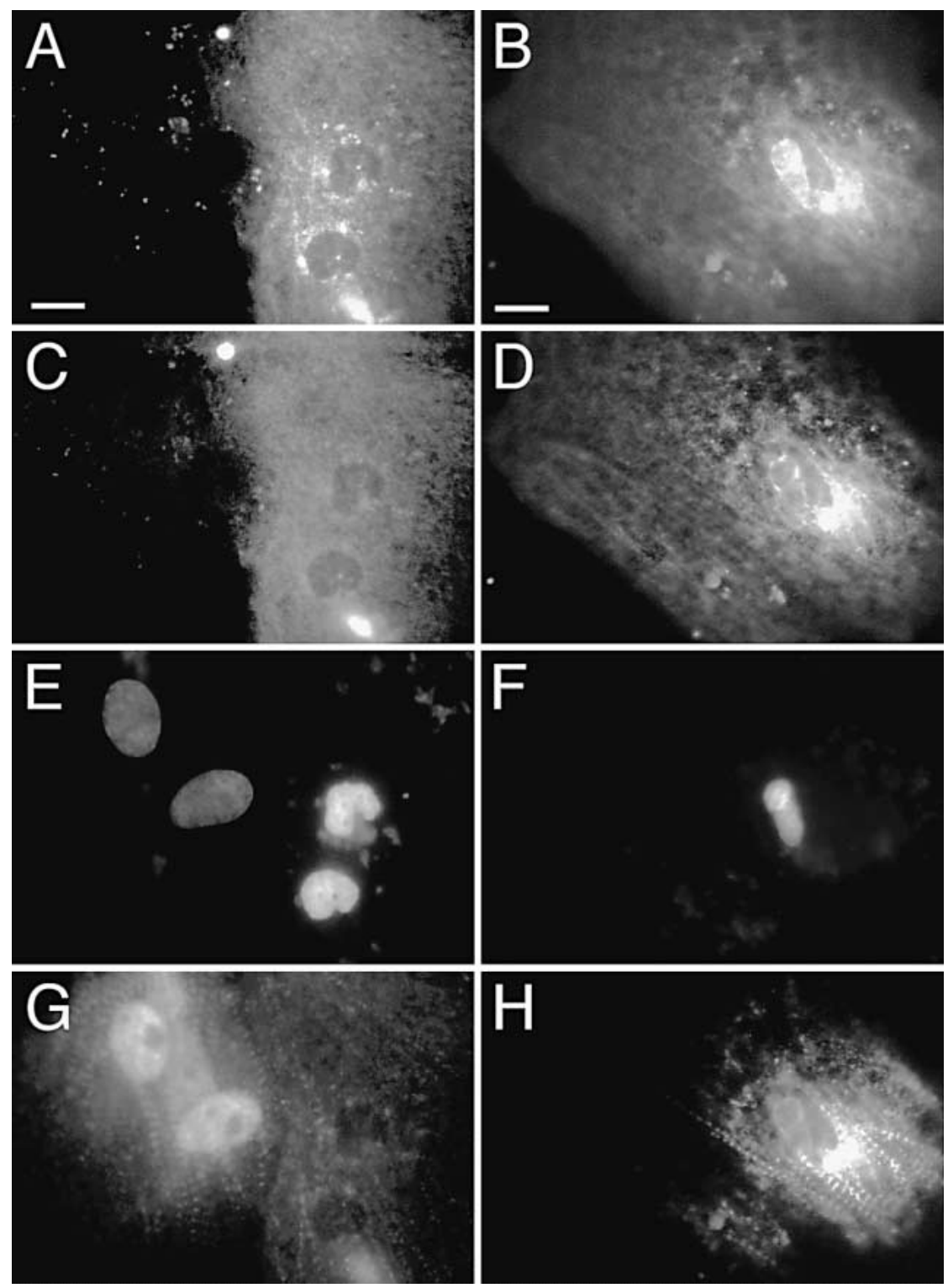

hand, an inhibitory mechanism could have been fully active. However, ectopic expression of wild-type versions of cyclin B1 and wild-type Cdc2 was sufficient to induce MPF activity in cultured ARC. This outcome may be due to a threshold effect in which exogenous cyclin B1-HA/ Cdc2-FLAG gradually titrated out a G2 phase inhibitor that was possibly in part responsible for the negative outcome when cyclin B1-HA was expressed alone.

Chromatin compaction was the most obvious effect of the simultaneous expression of cyclin B1-HA and Cdc2FLAG/Cdc2AF-FLAG. In practically all observed cases, nuclear condensation was encountered indicating a mitotic arrest; however, a proper segregation of chromatids could not be detected. The abnormally clumped morphology of chromosomes observed in ARC transfected with either pcDNA3.1cycB1-HA/cdc2-FLAG or pcDNA3. $1 \mathrm{cycB} 1-\mathrm{HA} / \mathrm{cdc} 2 \mathrm{AF}-\mathrm{FLAG}$ points to an induction by MPF, however, causing an inappropriately induced nuclei condensation by MPF. The diploid nuclear DNA content of the two nuclei in ARC is not prepared for a regular $M$ phase, because no DNA replication had previously taken place to generate two sister chromatids from each chromosome. This could result in a mitotic catastrophe followed by apoptotic cell death.

The observed chromatin compaction was accompanied by histone $\mathrm{H} 3$ phosphorylation revealing the po- 
Fig. 6. Histone $\mathrm{H} 3$ phosphorylation in cultured ARC. Cardiomyocytes were analyzed $72 \mathrm{~h}$ after transfection with pcDNA3.1cycB1-HA/cdc2-FLAG (A, C, E, G) and pHM6-lacZ encoding HA-tagged lacZ (B, D, F, H). Transfectants were identified by anti-HA antibody staining (A, B), and phosphorylation status of histone $\mathrm{H} 3$ was determined by anti-phospho-histone $\mathrm{H} 3$ antibody staining (C, D). DAPI was used to detect morphologic alterations in the nuclei shape (E, F), and the contractile apparatus was visualized by anti-myomesin antibody staining (G, H). Highly compact nuclei (E) were observed in transfectants identified by cyclin B1-HA expression (A). Condensation was associated with phosphorylation of histone $\mathrm{H} 3$ (c). Control transfection with pHM6-lacZ excluded potential artifacts: ARC expressing $\beta$-galactosidase (B) did not stain positive for H3 phosphorylation (D; extended exposure time); no chromosome condensation was seen $(\mathbf{F})$. Scale bar: $10 \mu \mathrm{m}$.
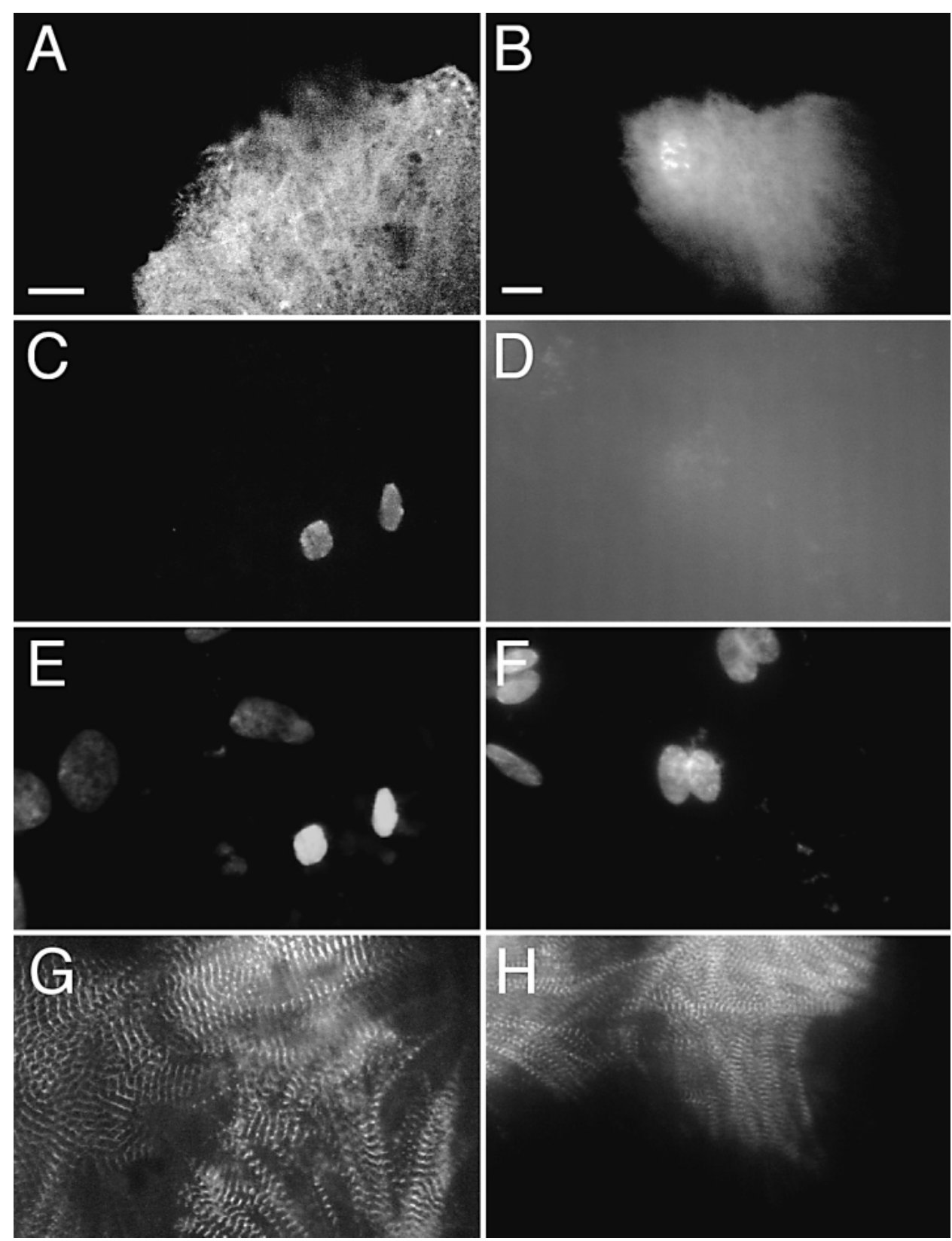

tential of MPF to initiate a typical cell cycle-related event. Apparently reconstitution of MPF made it possible for ARC, at least in part, to proceed in the cell cycle, whereby the histone $\mathrm{H} 3$ phosphorylation demonstrated activation of the corresponding kinase through MPF. The kinase responsible for histone $\mathrm{H} 3$ phosphorylation has been recently identified as the B-type aurora kinase in drosophila [Adams et al., 2001; Murnion et al., 2001], while such a kinase has not yet been identified in mammalian cells. Whether MPF in ARC is a direct regulator of the B-type aurora kinase activity cannot be judged at present.

MPF-induced condensation of the nuclei was accompanied by visible myofibrillar disorganization and decay and the occurrence of a disorganized contractile apparatus might be a consequence of the reinduced MPF activity. The function of cell cycle proteins stimulating cell cycle progression seems to be 'poisonous' for postmitotic cardiomyocytes in general. One reason for this phenomenon might be the acute problem for cardiomyocytes to 
undergo cell division and at the same time maintaining heart muscle contraction. The results obtained here as well as other reports showing that ectopic expression of other cell cycle components induces cardiomyocyte death [Kirshenbaum and Schneider, 1995; Liu and Kitsis, 1996; Agah et al., 1997; Akli et al., 1999; von Harsdorf et al., 1999] would clearly point in such a direction. A recent report emphasizes further the cytotoxic role of the cell cycle in cardiomyocytes, suggesting a role of cyclin-dependent kinase Cdk2 as the mediator in hypoxia-induced apoptosis [Adachi et al., 2001].

Evidently, resetting the cardiac cell cycle by forced expression of MPF at the G2/M cell cycle checkpoint led to chromosome condensation associated with histone $\mathrm{H} 3$ phosphorylation, whereas no chromosome segregation and cytokinesis were observed. While partial reentry into the cell cycle clearly could be observed, an impact of reconstituted MPF on the cells was limited in respect to cell cycle progression.

\section{Acknowledgements}

The authors would like to thank Vincenzo Busceti for technical assistance. We are grateful to Dr. Elisabeth Ehler for providing expertise in immunocytochemistry analysis, Prof. Dr. Jean-Claude Perriard and Dr. Monika Eppenberger-Eberhardt for helpful discussions. This work was supported by the Swiss Priority Program for Biotechnology of the Swiss National Science Foundation (NF-SPP 5002-46083).

\section{References}

Adachi, S., H. Ito, M. Tamamori-Adachi, Y. Ono, T. Nozato, S. Abe, M. Ikeda, F. Marumo, M. Hiroe (2001) Cyclin A/cdk2 activation is involved in hypoxia-induced apoptosis in cardiomyocytes. Circ Res 88: 408-414.

Adams, R.R., H. Maiato, W.C. Earnshaw, M. Carmena (2001) Essential roles of Drosophila inner centromere protein (INCENP) and aurora $\mathrm{B}$ in histone $\mathrm{H} 3$ phosphorylation, metaphase chromosome alignment, kinetochore disjunction, and chromosome segregation. J Cell Biol 153: 865-880.

Agah, R., L.A. Kirshenbaum, M. Abdellatif, L.D. Truong, S. Chakraborty, L.H. Michael, M.D. Schneider (1997) Adenoviral delivery of E2F-1 directs cell cycle reentry and p53-independent apoptosis in postmitotic adult myocardium in vivo. J Clin Invest 100: 2722-2728.

Akli, S., S. Zhan, M. Abdellatif, M.D. Schneider (1999) E1A can provoke G1 exit that is refractory to $\mathrm{p} 21$ and independent of activating cdk2. Circ Res 85: 319-328.

Brooks, G., R.A. Poolman, C.J. McGill, J.M. Li (1997) Expression and activities of cyclins and cyclin-dependent kinases in developing rat ventricular myocytes. J Mol Cell Cardiol 29. 2261-2271.

Cotten, M., E. Wagner, K. Zatloukal, S. Phillips, D.T. Curiel, M.L. Birnstiel (1992) High-efficiency receptor-mediated delivery of small and large (48 kilobase) gene constructs using the endosome-disruption activity of defective or chemically inactivated adenovirus particles. Proc Natl Acad Sci USA 89: 6094-6098.

Dätwyler, D.A., H.M. Eppenberger, D. Koller, J.E. Bailey, J.P. Magyar (1999) Efficient gene delivery into adult cardiomyocytes by recombinant Sindbis virus. J Mol Med 77: 859-864.
Dätwyler, D.A., J.P. Magyar, V. Busceti, A. Hirschy, J.C. Perriard, J.E. Bailey, H.M. Eppenberger (2001) Recombinant Sindbis virus allows expression and precise targeting of proteins of the contractile apparatus in cultured cardiomyocytes. Basic Res Cardiol 96: 630635.

Dirks, W., M. Wirth, H. Hauser (1993) Dicistronic transcription units for gene expression in mammalian cells. Gene 128: 247-249.

Eppenberger-Eberhardt, M., I. Flamme, V. Kurer, H.M. Eppenberger (1990) Reexpression of alpha-smooth muscle actin isoform in cultured adult rat cardiomyocytes. Dev Biol 139: 269_ 278.

Grove, B.K., V. Kurer, C. Lehner, T.C. Doetschman, J.C. Perriard, H.M. Eppenberger (1984) A new 185,000-dalton skeletal muscle protein detected by monoclonal antibodies. J Cell Biol 98: 518-524.

Hendzel, M.J., Y. Wei, M.A. Mancini, A. Van Hooser, T. Ranalli, B.R. Brinkley, D.P. BazettJones, C.D. Allis (1997) Mitosis-specific phosphorylation of histone $\mathrm{H} 3$ initiates primarily within pericentromeric heterochromatin during G2 and spreads in an ordered fashion coincident with mitotic chromosome condensation. Chromosoma 106: 348-360.

Huber, M., A. Limat, E. Wagner, D. Hohl (2000) Efficient in vitro transfection of human keratinocytes with an adenovirus-enhanced receptormediated system. J Invest Dermatol 114: 661666.

Kang, M.J., J.S. Kim, S.W. Chae, K.N. Koh, G.Y. Koh (1997) Cyclins and cyclin dependent kinases during cardiac development. Mol Cells 7: 360-366.
Kass-Eisler, A., E. Falck-Pedersen, M. Alvira, J. Rivera, P.M. Buttrick, B.A. Wittenberg, L. Cipriani, L.A. Leinwand (1993) Quantitative determination of adenovirus-mediated gene delivery to rat cardiac myocytes in vitro and in vivo. Proc Natl Acad Sci USA 90: 1149811502.

Kirshenbaum, L.A., W.R. MacLellan, W. Mazur, B.A. French, M.D. Schneider (1993) Highly efficient gene transfer into adult ventricular myocytes by recombinant adenovirus. J Clin Invest 92: 381-387.

Kirshenbaum, L.A., M.D. Schneider (1995) Adenovirus E1A represses cardiac gene transcription and reactivates DNA synthesis in ventricular myocytes, via alternative pocket proteinand p300-binding domains. J Biol Chem 270: 7791-7794.

Koshland, D., A. Strunnikov (1996) Mitotic chromosome condensation. Annu Rev Cell Dev Biol 12: 305-333.

Krek, W., E.A. Nigg (1991) Mutations of p34cdc2 phosphorylation sites induce premature mitotic events in HeLa cells: Evidence for a double block to $\mathrm{p} 34 \mathrm{cdc} 2$ kinase activation in vertebrates. EMBO J 10: 3331-3341.

Liao, H.S., P.M. Kang, H. Nagashima, N. Yamasaki, A. Usheva, B. Ding, B.H. Lorell, S. Izumo (2001) Cardiac-specific overexpression of cyclin-dependent kinase 2 increases smaller mononuclear cardiomyocytes. Circ Res 88: 443-450.

Liu, Y., R.N. Kitsis (1996) Induction of DNA synthesis and apoptosis in cardiac myocytes by E1A oncoprotein. J Cell Biol 133: 325-334.

Murnion, M.E., R.R. Adams, D.M. Callister, C.D. Allis, W.C. Earnshaw, J.R. Swedlow (2001) Chromatin-associated protein phosphatase 1 regulates aurora-B and histone $\mathrm{H} 3$ phosphorylation. J Biol Chem 276: 26656-26665. 
Nurse, P. (1990) Universal control mechanism regulating onset of M-phase. Nature 344: 503508.

Obermann, W.M., M. Gautel, F. Steiner, P.F. van der Ven, K. Weber, D.O. Furst (1996) The structure of the sarcomeric $\mathrm{M}$ band: Localization of defined domains of myomesin, M-protein, and the $250-\mathrm{kD}$ carboxy-terminal region of titin by immunoelectron microscopy. J Cell Biol 134: 1441-1453.

Poolman, R.A., G. Brooks (1998) Expressions and activities of cell cycle regulatory molecules during the transition from myocyte hyperplasia to hypertrophy. J Mol Cell Cardiol 30: 21212135.

Rust, E.M., M.V. Westfall, J.M. Metzger (1998) Stability of the contractile assembly and $\mathrm{Ca}^{2+}$ activated tension in adenovirus infected adult cardiac myocytes. Mol Cell Biochem 181: 143155.
Stein, G.S., J.L. Stein, J.B. Lian, T.J. Last, T.A. Owen, L. McCabe (1998) Synchronization of normal diploid and transformed mammalian cells; in Celis, J.E. (ed): Cell Biology: A Laboratory Handbook, ed 2. San Diego, Academic Press, pp 253-260.

von Harsdorf, R., L. Hauck, F. Mehrhof, U. Wegenka, M.C. Cardoso, R. Dietz (1999) E2F-1 overexpression in cardiomyocytes induces downregulation of p21CIP1 and p27KIP1 and release of active cyclin-dependent kinases in the presence of insulin-like growth factor I. Circ Res 85: 128-136.

Wagner, E., K. Zatloukal, M. Cotten, H. Kirlappos, K. Mechtler, D.T. Curiel, M.L. Birnstiel (1992) Coupling of adenovirus to transferrin-polylysine/DNA complexes greatly enhances receptor-mediated gene delivery and expression of transfected genes. Proc Natl Acad Sci USA 89: 6099-6103.
Wei, S.K., H.M. Colecraft, C.D. DeMaria, B.Z. Peterson, R. Zhang, T.A. Kohout, T.B. Rogers, D.T. Yue (2000) $\mathrm{Ca}(2+)$ channel modulation by recombinant auxiliary beta subunits expressed in young adult heart cells. Circ Res 86 . 175-184.

Westfall, M.V., E.M. Rust, F. Albayya, J.M. Metzger (1997) Adenovirus-mediated myofilament gene transfer into adult cardiac myocytes. Methods Cell Biol 52: 307-322.

Wightman, L., E. Patzelt, E. Wagner, R. Kircheis (1999) Development of transferrin-polycation/ DNA-based vectors for gene delivery to melanoma cells. J Drug Target 7: 293-303. 